\title{
How selecting best therapy for metastatic NTRK fusion-positive non-small cell lung cancer?
}

\author{
Simon Ekman^ \\ Thoracic Oncology Center, Karolinska University Hospital/Department of Oncology-Pathology, Karolinska Institutet, Stockholm, Sweden \\ Correspondence to: Simon Ekman, MD, PhD. Thoracic Oncology Center, Karolinska University Hospital/Department of Oncology-Pathology, \\ Karolinska Institutet, S-17176 Stockholm, Sweden. Email: simon.ekman@ki.se.
}

\begin{abstract}
The tropomyosin receptor kinase (TRK) family of receptor tyrosine kinases has become a focus of clinical interest because the NTRK genes (NTRK1-3) encoding them have been identified as oncogenic fusion genes in a wide range of different tumor types, including lung cancer. These NTRK gene fusions usually occur at a low frequency below $1 \%$, in non-small cell lung cancer (NSCLC) in $0.1-0.2 \%$ of the cases and have been reported across a wide range of tumor types. The TRK fusion proteins encoded by such gene fusions have constitutively activated tyrosine kinase domains and constitute actionable targets for tyrosine kinase inhibitors (TKIs). The first generation TRK TKIs larotrectinib and entrectinib have been investigated in clinical phase I and II trials in solid tumors both in adult and pediatric patients and results have demonstrated high response rates that are durable and with generally good tolerability. This has led to approval of these TRK inhibitors by regulatory authorities in the USA, Europe and Japan as tumor agnostic treatment of advanced or recurrent NTRK fusion-positive cancers in adult and pediatric patients. With a focus on lung cancer, this review gives a background to NTRK fusion genes, presents clinical data for TRK inhibitors and discuss the issue of acquired resistance to TRK inhibition.
\end{abstract}

Keywords: NTRK; tropomyosin receptor kinase (TRK); fusion; non-small cell lung cancer (NSCLC); inhibitor

Submitted Mar 15, 2020. Accepted for publication Sep 25, 2020.

doi: $10.21037 /$ tlcr-20-434

View this article at: http://dx.doi.org/10.21037/tlcr-20-434

\section{Introduction}

Lung cancer is one of the most common cancer types with around 2 million new cases diagnosed globally each year and by far the most deadly cancer type with around 1,76 million lung cancer deaths each year in the world (1). With the advent of precision cancer medicine targeting oncogenic drivers the treatment landscape of non-small cell lung cancer (NSCLC) has drastically changed with improved survival and quality-of-life for patients treated with tyrosine kinase inhibitors (TKIs) against various oncogenic genetic aberrations. The identification of gene fusions as oncogenic drivers in cancer in general and in NSCLC in particular has led to important developments of novel diagnostic techniques and therapeutics in these fusion gene positive cancers. Interestingly, in the search of oncogenic fusion genes it has been discovered that many of these occur across other tumor types than lung cancer. In NSCLC, the identification of the gene rearrangement between Echinoderm microtubule-associated proteinlike 4 (EML4) and the anaplastic lymphoma kinase (ALK) in a NSCLC patient in 2007 (2) started a new era of treating oncogenic gene fusions in NSCLC with TKIs. Later, rearrangements of the gene encoding ROS1 protooncogene receptor tyrosine kinase was identified as a molecular subgroup of NSCLC highly susceptible to the TKI crizotinib (3). Both ALK and ROS1 are now clinically actionable targets tested for and treated with TKIs in clinical routine.

\footnotetext{
$\wedge$ ORCID: 0000-0002-8343-6226.
} 
The tropomyosin receptor kinase (TRK) family of receptor tyrosine kinases (RTKs) has recently become a focus of interest in the clinical setting because the NTRK genes encoding them have been identified as oncogenic fusion genes in a wide range of different tumor types, including lung cancer. This review gives a background to $N T R K$ genes in cancer and describes the recent clinical developments in targeting NTRK fusion gene positive lung cancer.

\section{NTRK genes and TRK receptors}

The RTKs TRKA, TRKB and TRKC are transmembrane receptor tyrosine kinases encoded by the neurotrophic TRK (NTRK) genes, where TRKA is encoded by NTRK1 located on chromosome 1q21-q22, TRKB by NTRK2 located on chromosome 9q22.1 and TRKC by NTRK3 on chromosome $15 \mathrm{q} 25$ (4). Structurally, the TRK receptors consist of an extracellular domain, a transmembrane region and an intracellular part containing the tyrosine kinase domain. The extracellular domain contains a cysteine-rich cluster (C1), three leucine-rich 24-residue repeats (LRR1-3), another cysteine-rich cluster (C2) and two immunoglobulin-like domains (Ig1 and Ig2). TRK receptors are activated by a family of ligands called the neurotrophins that are involved in multiple ways in the development and function of the central nervous system. Of the four neurotrophins identified, Nerve Growth Factor (NGF) binds to TRKA, brain-derived neurotrophic factor (BDNF) and neurotrophin 4 (NT-4) bind to TRKB, and neurotrophin 3 (NT-3) binds to TRKC (5).

Upon ligand binding and receptor dimerization, the TRK receptors undergo autophosphorylation of tyrosine residues in the intracellular domain leading to activation of downstream signaling pathways, including Ras/Raf/MAPK, $\mathrm{PI} 3 \mathrm{~K} / \mathrm{Akt} / \mathrm{mTOR}$ and PLC $\gamma$ pathways. In this way, the TRK receptors regulate different cellular processes such as proliferation, survival and differentiation. The TRK receptor signaling pathways play crucial roles in neuronal differentiation and development and in maintaining normal neuronal homeostasis. Their activation has been shown to be involved in synapse formation and plasticity as well as axon and dendrite formation, with implications in memory function, nociception and proprioception (6). Neurotrophins and their TRK receptors also have roles in non-neuronal tissue including skeletal tissue, vasculature, and the immune system (7-9). In human lung tissue, neurotrophins and their receptors have been showed to be expressed on a protein level in different components of the lung, including bronchial epithelial cells and alveolar cells, suggesting a role in the regulation of normal lung function (10).

\section{Discovery of NTRK gene fusions in cancer}

Constitutive activation of the TRK receptors can occur through different chromosomal rearrangements, including inversions, deletions or translocations, resulting in in-frame fusions of the C-terminal tyrosine kinase domain of the NTRK genes with an $\mathrm{N}$-terminal fusion partner. Generally, the fusion eliminates the ligand binding site, resulting in ligand-independent dimerization and phosphorylation of the receptors (Figure 1). The majority of the $5^{\prime}$ fusion partners described contains a gene sequence encoding one or more dimerization domains that mediate this ligand-independent activation. The first NTRK fusion gene observed was in a patient with colorectal cancer in 1986 involving the tropomyosin 3 gene (TPM3) and NTRK1 (11). Later on other NTRK fusions have been discovered in colorectal cancer, including LMNA-NTRK1 and SCYL3-NTRK1 $(12,13)$. Infantile fibrosarcoma, which is a rare malignant tumor of fibroblasts representing $<1 \%$ of pediatric cancers, has been characterized by an ETV6-NTRK3 fusion gene in around $70 \%$ of the cases, involving a translocation of chromosomes 12 and 15 (14). The same fusion gene has also been reported as commonly occurring in the rare secretory carcinomas of the breast (15) and salivary gland (16). NTRK fusion genes have also been described in multiple other tumor types, including in papillary thyroid carcinoma, especially radiation-associated, lung cancer, gliomas, other sarcomas, inflammatory myofibroblastic tumors, melanocytic tumors, and intrahepatic cholangiocarcinomas (5). Up to now, more than 80 different fusion partners to NTRK have been identified in many different adult and pediatric tumor types. Generally, NTRK fusions are found at low frequencies in common tumor types (commonly $<1 \%$ ) whereas in some rare cancers they occur at a high frequency (>80\%). Fusion genes involving NTRK1 and NTRK3 appear to occur more commonly, including in thyroid cancer, whereas NTRK2 fusions occur in some cancer types, such as gliomas and sarcomas (17).

\section{TRK receptors/neurotrophins and NTRK fusion genes in lung cancer}

Neurotrophins and their TRK receptors have been implicated in lung cancer in several studies. Preclinical 


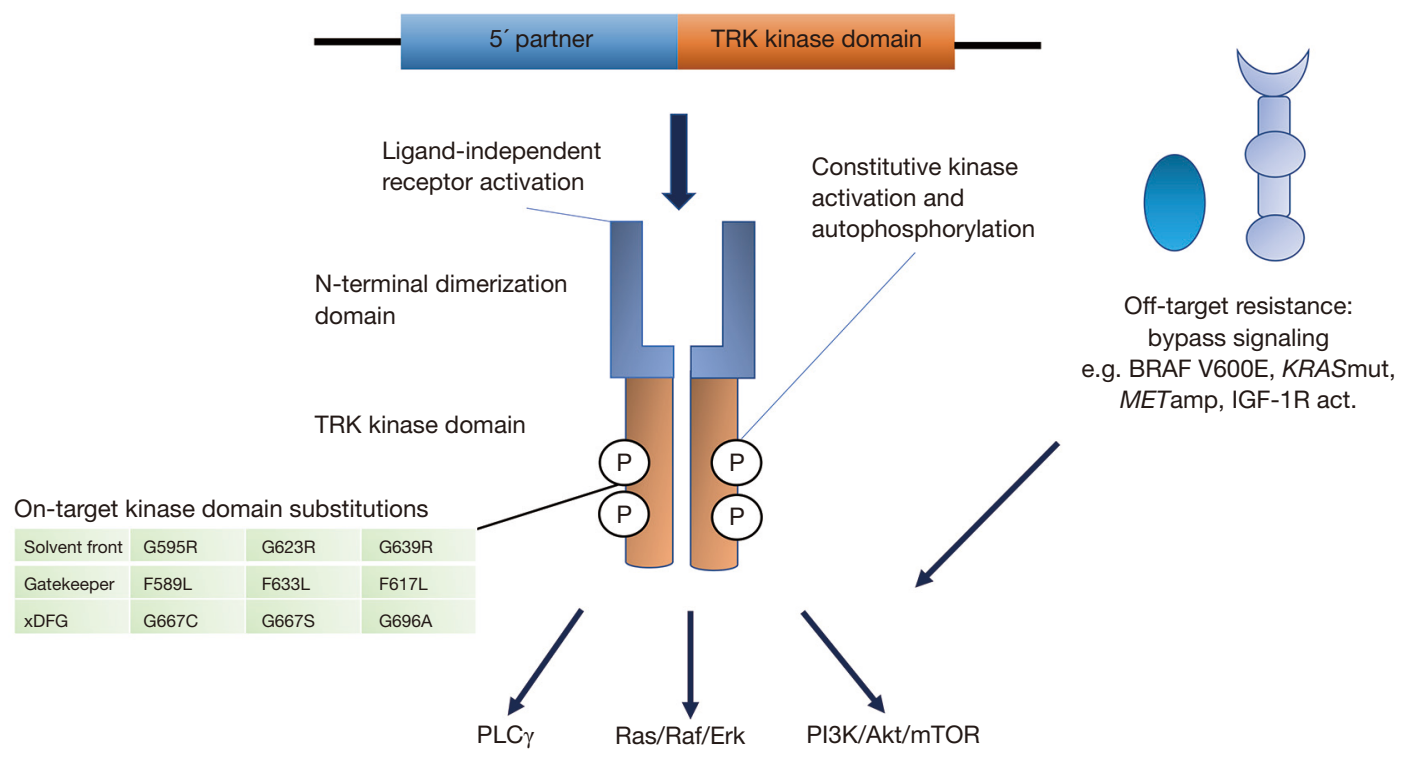

Figure 1 Constitutive activation of the TRK receptors occurs through different chromosomal rearrangements resulting in in-frame fusions of the C-terminal tyrosine kinase domain of the NTRK genes with an N-terminal fusion partner. The fusion results in ligand-independent dimerisation and autophosphorylation of the receptors, leading to constitutive activation of downstream signaling pathways, including Ras/ Raf/Erk, PI3K/Akt/mTOR and PLC $\gamma$ pathways. Acquired resistance to TRK inhibitors are mediated by either on-target substitutions in the kinase domain (in solvent front, gatekeeper region or xDFG motif) or off-target activation of bypass signaling pathways. KRASmut, KRAS mutation; MET amp, MET amplification; IGF-1R act, IGF-1R activation.

studies in lung cancer cell lines and a mouse model identified $\mathrm{BDNF}$ and $\operatorname{TrkB}$ to be important for migration and metastasis, where knockdown of TrkB in human lung cancer cell lines significantly decreased migration and metastasis. TrkB protein expression in patient samples was also found to be correlated with distant metastasis (18). Another study demonstrated that TrkB and BDNF protein expression determined by immunohistochemistry (IHC) was significantly correlated with clinical outcomes, where TrkBpositive tumors had significantly worse disease-free survival and overall survival compared to TrkB-negative tumors, and that co-expression of TrkB and BDNF resulted in a poorer prognosis compared with overexpression of either protein alone (19). Odate et al. investigated the protein expressions of TrkB and BDNF analyzed by IHC in surgical samples of lung cancer $(n=104)$ and expressions were significantly higher in neuroendocrine tumors (NETs) compared with non-NETs, in particular in large cell neuroendocrine carcinomas (LCNEC) (20).

Rearrangements of NTRK occur in a small subgroup of lung cancer $(<1 \%)$. The first NTRK gene rearrangements described in lung cancer was in 2013 in a fraction of
NSCLC patients with adenocarcinoma histology. In this study, targeted next generation sequencing (NGS) was performed in tumor samples from 36 patients without any known genetic alterations using standard clinical assays. NTRK1 fusion genes were observed in 3/91 (3.3\%) of patients, including two novel fusion partners, myosin phosphatase Rho-interacting protein gene (MPRIP) and CD74, respectively. In addition, a novel TMP53-NTRK2 rearrangement was also reported (21). In a phase I study of entrectinib in NTRK-rearranged solid tumors, 1,378 NSCLC patients were screened and one patient with lung adenocarcinoma harbored a novel SQSTM1-NTRK1 fusion gene and one patient had a previously described TPM3NTRK1 rearrangement, resulting in an NTRK fusion gene incidence of $0.1 \%$ (22). NTRK fusions involving NTRK2 has also been reported with a TRIM24-NTRK2 gene fusion identified in a lung adenocarcinoma in RNAseq data from the Cancer Genome Atlas (17). In a study by Farago et al., 11 NSCLC patients harboring NTRK gene fusions verified by NGS were described (23). NTRK fusions involved NTRK1 in 7 cases and NTRK3 in 4 cases, the latter including fusion partners ETV6 and SQSTM1. The patients 
described in this cohort had no other concurrent oncogenic drivers identified, $55 \%$ were male, median age at diagnosis was 47.6 years and smoking status with a median pack year history of 0 (range, 0-58). Regarding histology, nine cases were adenocarcinoma, one was squamous cell carcinoma and one neuroendocrine carcinoma. In 4,872 consecutively screened NSCLC patients, the estimated frequency of NTRK fusions in NSCLC was $0.23 \%$. The discrepancy of reported incidence of NTRK fusions in NSCLC between different studies is likely reflecting the different patient populations and sample sizes, but the incidence is likely to be low in the range of $0.1-0.2 \%$.

\section{Methods for detection of NTRK fusion genes}

There are several different methods for the diagnosis of NTRK fusions (24). These methods include DNA-based or RNA-based NGS, fluorescence in-situ hybridization (FISH) or IHC (25). Clinical testing of NTRK fusions has been dominated by NGS. However, not all NGS platforms are optimized to identify NTRK fusions and especially the detection of NTRK2 and NTRK3 may be complicated by the presence of large intronic regions. Targeted RNA sequencing can be a complementary method to DNAbased NGS assays and enables detection of both known and unknown upstream gene partners. Besides tissue-based testing of DNA or RNA, NTRK fusions can potentially be detected using blood-based cell-free DNA (cfDNA) testing. FISH and RT-PCR have been successfully used in the clinic to detect oncogenic fusions in solid tumors and are especially useful for tumor types with a high prevalence of known NTRK fusions, such as ETV6-NTRK3 in infantile fibrosarcomas, secretory breast carcinoma, and mammary analogue secretory carcinoma (MASC). Advantages of FISH include the low amount of material needed (a few unstained slides) and that it can be performed relatively quickly within a few days. IHC to examine protein expression is another method that can be used for the detection of TRK overexpression reflecting the potential presence of an NTRK fusion. IHC has several advantages: it is commonly used in clinical pathology laboratories, it is inexpensive, requires limited amount of material and has a fast turnaround time. The sensitivity and specificity of pan-TRK antibodies have shown to be acceptable with a sensitivity ranging from $75 \%$ to more than $96 \%$ and a specificity ranging from $92 \%$ to $100 \%$, however these being variable depending on NTRK fusion type and tumor type (26,27). With limited resources and/or access to NGS, using IHC as a screening tool for
NTRK fusions is a valid option, but confirming positive IHC stainings with nucleic acid-based testing is needed. On the other hand, IHC should be used to confirm protein expression of NTRK fusions detected by nucleic acid-based testing since not all NTRK fusion genes are expressed and the protein kinase is the pharmacological target (27).

\section{TRK inhibitors in treatment of NTRK-positive cancer including NSCLC}

\section{First-generation TRK inbibitors}

There are two first generation TRK TKIs, larotrectinib and entrectinib, which potently and selectively inhibit TRKA, TRKB and TRKC, with IC50 values ranging between 5-11 and $1-5 \mathrm{nM}$, respectively, for TRKA/B/C in in vitro assays $(28,29)$. Whereas larotrectinib is a selective inhibitor of TRKA/B/C, entrectinib in addition inhibits ROS1 (IC50 $0.2 \mathrm{nM}$ ) and ALK (IC50 1.6 nM) (30) (Table 1). There is also a group of multi-kinase inhibitors that inhibits the TRK receptors, including inhibitors that are approved for other indications such as crizotinib, cabozantinib, ponatinib and nintedanib, as well as the investigational inhibitors lestaurtinib, altiratinib, foretinib, merestinib, MGCD516, PLX7486, DS-6051b, and TSR-011 (6). These multi-kinase inhibitors have not been well characterized in the clinical setting compared to the more selective TKIs.

\section{Larotrectinib}

Larotrectinib has been clinically investigated regarding safety and efficacy in patients harboring NTRK-rearranged tumors in three clinical trials; a phase 1 study involving adults, a phase 1-2 study involving children (SCOUT), and a phase 2 study involving adolescents and adults (NAVIGATE) (Table 2).

There have been several reports from these studies (28,31). Responses have been reported to occur regardless of tumor type and treatment considered tolerable with the majority of adverse events (93\%) being grade 1 or 2 . The most common adverse events included anemia (11\%), increased liver enzymes (7\%), weight increase $(7 \%)$, and decrease in neutrophil count $(7 \%)$. Other adverse events reported include fatigue, dizziness, nausea and constipation. Recently, a pooled analysis of the three trials was published with 159 TRK fusion-positive cancer patients (32). Objective response rate was reported to be $79 \%$ according to investigator assessment, with $16 \%$ CR. Twelve patients with lung cancer were included, 9 of whom $(75 \%)$ had an 
Table 1 Kinase activity of TRK inhibitors in clinical use and under development

\begin{tabular}{lccc}
\hline Kinase inhibitor: kinase & Larotrectinib & Entrectinib & Selitrectinib \\
\hline TRKA/B/C & + & + & + \\
ROS1 & & + & Repotrectinib \\
ALK & & + & + \\
TRKA/B/C on-target mutations & & + & + \\
\hline
\end{tabular}

TRK, tropomyosin receptor kinase.

Table 2 TRK inhibitors in selected clinical studies

\begin{tabular}{|c|c|c|c|c|c|c|c|}
\hline Study/author & Treatment & Design & No. of pts. & ORR (\%) & PFS (months) & OS (months) & trAEs (\% gr.3-5) \\
\hline Lassen et al. (31) & Larotrectinib & Phase 1/2 & 122 & 81 & NA & NA & $\begin{array}{c}\text { gr.3: } 6 \%+<1 \% \text { for fatigue, dizziness, } \\
\text { myalgia, gr.4:<1\% ALT increase, gr.5: } \\
0 \%\end{array}$ \\
\hline Hong et al. (32) & Larotrectinib & Phase 1/2 & 159 & 79 & 28.3 (22.1-NE) & 44.4 (36.5-NE) & gr.3: $39 \%$, gr.4: $7 \%$, gr.5: $0 \%$ \\
\hline Doebele et al. (34) & Entrectinib & Phase 1/2 & 54 & 57 & $11.2(8.0-14.9)$ & $\begin{array}{c}\text { Estimated } 21 \\
(14.9-\mathrm{NE})\end{array}$ & gr.3: $61 \%$, gr.4: $9 \%$, gr.5: $0 \%$ \\
\hline Hyman et al. (35) & Selitrectinib & Phase I + EAP & 29 & 34 & NA & NA & \\
\hline
\end{tabular}

In brackets for PFS and OS, 95\% CI. TRK, tropomyosin receptor kinase; ORR, overall response rate; PFS, progression-free survival; OS, overall survival; NA, not available; EAP, Early Access Program; trAEs, treatment-related adverse events; gr., grade; Cl, confidence interval; $\mathrm{NE}$, not estimable.

objective response. There were 12 evaluable patients with brain metastases before enrollment with 9 of them $(75 \%)$ having a response. Only 3 of the 12 patients with brain metastases had measurable disease at baseline; of these one had a CR, one a PR and one stable disease. The most common grade 3 or 4 larotrectinib-related adverse events were increased alanine aminotransferase (3\%), anaemia $(2 \%)$, and decreased neutrophil count $(2 \%)$. The most common larotrectinib-related serious adverse events were increased alanine aminotransferase $(2 / 260$ patients, $<1 \%)$, increased aspartate aminotransferase $(<1 \%)$, and nausea $(<1 \%)$. No grade $5 \mathrm{AE}$ occurred. A recent update from ESMO 2020 on 14 lung cancer patients (13 with NSCLC and one with small cell lung cancer) reported an ORR of $71 \%$ (1 CR, 9 PR) and with a median PFS not reached, estimated PFS rate at 12 months was $69 \%$. The ORR in patients with CNS metastases was 57\% (36).

The results from the clinical trials described above lead to the approval of larotrectinib by the Food and Drug Administration (FDA) in November 2018 for adult and pediatric patients with solid tumors that have a NTRK gene fusion, that are either metastatic or where surgical resection is likely to result in severe morbidity, and who have no satisfactory alternative treatments or whose cancer has progressed following treatment. A similar approval was granted in July 2019 by the European Medicines Agency (EMA) being the first histology-independent agnostic cancer treatment approved in Europe.

\section{Entrectinib}

The clinical trials that have led the clinical development of entrectinib are a phase I trial in adults (ALKA-372-001, Italy), a separate adult phase I trial (STARTRK-1, global), a phase II basket trial (STARTRK-2, including NTRK1/2/3, ROS1 or $A L K$ gene fusions), and a phase $\mathrm{I} / \mathrm{Ib}$ pediatric trial (STARTRK-NG) (Table 1). Demetri and colleagues first reported on efficacy in 54 adult patients included in these trials and safety results reported in 355 patients (33). The most common tumor types were sarcomas (24\%), lung cancer (19\%), mammary analogue secretory carcinoma $(13 \%)$ and breast cancer $(11 \%)$. In the safety population, most treatment-related AEs were grade 1-2 and managed 
with dose reduction in $27.3 \%$. The most common AEs included dysgeusia, constipation, fatigue, diarrhoea, oedema and dizziness. A recent integrated analysis of three phase $1 / 2$ clinical trials enrolling adult patients with metastatic or locally advanced NTRK fusion-positive solid tumors who received entrectinib was reported (34). These studies are ongoing; NCT02097810 (STARTRK-1), NCT02568267 (STARTRK-2), and EudraCT, 2012-000148-88 (ALKA372-001). Fifty-four patients were evaluable for efficacy representing ten different tumor types and 19 different histologies. Most patients had a NTRK1 or NTRK3 fusion with the most frequently represented gene fusion being ETV6-NTRK3, which was identified in $46 \%$ of the patients. Thirty-one of 54 patients had an ORR (57\%), of which $7 \%$ were CRs and $50 \%$ PRs. Median duration of response was 10 months and median PFS was 11 months. At data cutoff, the estimated median OS was 21 months (95\% CI 14.9 to not estimable). Twelve out of 54 patients (22\%) had baseline CNS metastases, as assessed by investigator, with a $50 \%$ partial response rate per blinded independent central review. In total, $55 \%$ of the brain metastasis patients had an intracranial response (6 out of 11) according to blinded independent review. Safety analysis was reported from two populations, including an NTRK fusion-positive population (68 patients from STARTRK-1, STARTRK-2, and ALKA372-001 who received at least one dose of entrectinib) and an overall safety-evaluable population (355 patients), including the phase 1 STARTRK-NG study with patients of any tumor type and gene rearrangement who received at least one dose of entrectinib. Consistent with the report from Demetri and colleagues mentioned above, the most common treatment-related AEs in both safety populations were grade 1-2 and reversible. The most common grade 3 or 4 treatment-related AEs in both safety populations were increased weight $(10 \%$ in the NTRK fusion-positive safety population and in $5 \%$ in the overall safety-evaluable population) and anaemia (12\% and 5\%, respectively). The most common serious treatment-related AEs were nervous system disorders (4\% and $3 \%$, respectively) and $4 \%$ of patients in both safety populations discontinued entrectinib due to treatment-related adverse events. No treatmentrelated deaths occurred. A recent update on the ALKA-372001, STARTRK-1 and STARTRK-2 studies with a focus on NSCLC patients $(n=13)$ demonstrated an ORR of $69 \%$, 9 patients had baseline CNS metastases showing an equally good ORR of $67 \%$ (37).

Based on the clinical trial data described above, FDA granted accelerated approval to entrectinib in August 2019 for adults and pediatric patients 12 years of age and older with solid tumors that have a NTRK gene fusion without a known acquired resistance mutation, are metastatic or where surgical resection is likely to result in severe morbidity, and have progressed following treatment or have no satisfactory standard therapy. Entrectinib has also received approval in Japan in June 2019 with the same indication as defined by FDA.

\section{TRK inbibitor resistance and next generation TRK inbibitors}

\section{On-target resistance}

Despite high clinical activity of TRK inhibitors, acquired resistance to treatment is a major clinical issue and cases of resistance to entrectinib and larotrectinib have been reported. This resistance can be mediated by on-target and off-target mechanisms (Figure 1). On-target resistance occurs via mutations in the NTRK kinase domain of the oncogenic fusion gene resulting in amino acid substitutions that involve three major regions: the solvent front, the gatekeeper residue or the activation loop xDFG motif. These alterations cause resistance by steric hindrance, altering the conformation of the kinase domain or changing ATP-binding affinity. The first reported case of acquired resistance to TRK inhibition occurred in a patient with LMNA-NTRK1 rearranged colorectal cancer after 4 months of entrectinib treatment (38). Sequencing of cfDNA revealed two mutations that affected the kinase domain and resulted in the two substitutions G595R and G667C. The G595R substitution is located in the solvent front region of the TRK kinase domain (paralogous to the ALK G1202R and ROS1 G2032R substitutions) and G667C is located in the ' $x$ ' position of the $x D F G$ motif in the activation segment of the TRKA kinase domain (paralogous to the ALK G1269A). Another solvent front substitution, G623R in TRKC, was reported in a patient with mammary analogue secretory carcinoma (MASC) progressing on entrectinib and this substitution is estimated to sterically interfere with drug binding (39). Furthermore, gatekeeper substitutions have been identified, including TRKA-F589L, TRKBF633L and TRKC-F617L that are paralogous to ALKL1196M and ROS1-L2026M (40). Substitutions involving the xDFG site of TRKA (G667S) and TRKC (G696A) have been identified in patients progressing on larotrectinib (28).

\section{Off-target resistance}

Off-target resistance to TRK TKIs may develop in a similar 
way as for other TKI treatments against oncogenic-driven cancer. This resistance is mediated by the activation of various parallel bypass signaling pathways circumventing the blockade by the on-target TKI. Recently, Cocco et al. reported on off-target resistance mechanisms in a series of TRK inhibitor-treated patients and patient-derived models mediated by genomic alterations that converged to activate the mitogen-activated protein kinase (MAPK) pathway (41). These alterations included a BRAF V600E mutation, KRAS mutations and a MET amplification. MAPK pathwaydirected targeted therapy, alone or in combination with TRK inhibition, managed to re-establish disease control. Another off-target resistance mechanism identified in cell line models is the activation of the insulin growth factor receptor type 1 (IGF-1R) pathway (42).

\section{Next generation TRK inhibitors}

Selitrectinib (LOXO-195, BAY2731954) and repotrectinib (TPX-0005) are the two major second generation TRK inhibitors in clinical development (Table 1) and that are able to inhibit most on-target NTRK resistance mutations while maintaining activity against wild-type TRKA/B/C. Both these inhibitors have demonstrated in vitro activity in the low nanomolar concentration range against both wild-type TRKA/B/C and many of the resistant TRK mutants (Table 2).

Clinical data on selitrectinib was reported from patients enrolled in a phase I clinical trial and a FDA expanded access program (35). All the patients, both adult and pediatric, had previously been treated with at least one firstgeneration TRK targeted agent. Twenty-five of the total 29 patients were evaluable for response and ten of the patients (34\%) had a confirmed complete or partial response. Nine of 20 patients (45\%) whose tumors had become resistant by secondary on-target NTRK gene mutations had a complete or partial response. None of the three patients whose tumors had become resistant by TRK-independent mechanisms responded to selitrectinib. The safety and efficacy of selitrectinib is currently being investigated in a phase I/II trial in adult and pediatric patients with NTRKrearranged cancers after prior treatment with a different TRK inhibitor (NCT03215511).

The clinical activity of repotrectinib has been reported from an ongoing phase I/II study of (TRIDENT-1; NCT03093116), in which a patient with MASC and acquired resistance to entrectinib mediated by a solvent front mutation (TRKA-G623E) had a response to repotrectinib (43). Another patient with a larotrectinib-resistant cholangiocarcinoma with LMNA-TRKA G595R and F589L mutations also experienced a tumor regression (44).

\section{Conclusions}

NTRK fusions are oncogenic drivers that have been identified in a wide variety of adult and pediatric cancers, in some cancers highly enriched and in others occurring at a low frequency, including in common cancers like lung cancer. Treatment with a first-generation TRK inhibitor results in high response rates irrespective of tumor type, fusion type and age. However, acquired resistance to TRK inhibitors is a clinical issue and occurs via two different mechanisms; on-target resistance mediated by TRK kinase domain substitutions or off-target activation of bypass signaling pathways. On-target resistance may be overcome with next generation TRK inhibitors that are currently undergoing clinical trials and sequential TRK targeted therapy may therefore be a treatment option for some NTRK fusion-positive patients. There is still scarce data about the optimal treatment sequence of TRK inhibitors in relation to other therapies, including chemotherapy and immune checkpoint inhibitors, and also the efficacy of these other treatments in NTRK fusion-positive NSCLC. In parallel, there is data indicating that immune checkpoint inhibitors in other oncogenic-driven lung tumors, including epidermal growth factor receptor (EGFR)-mutated and $A L K$ rearranged NSCLC have limited efficacy (45). Considering the high response rates of TRK inhibitors also in first line, and the scarcity of data for other therapies, it can be recommended that TRK inhibitors are used as first-line therapy if the NTRK fusion is discovered prior to the start of first-line systemic therapy. Upon progression, initial systemic therapy options used for other non-oncogenicdriven NSCLC can applied or alternatively inclusion into clinical trials with next generation TRK-inhibitors. If the NTRK fusion is discovered during first-line systemic therapy there is an option to complete the ongoing systemic therapy or interrupt the ongoing treatment and switch to a TRK-inhibitor depending on the clinical situation. These recommendations are also in line with the FDA and EMA approvals and the National Comprehensive Cancer Network (NCCN) guidelines. Generally, TRK inhibitors demonstrate a good tolerance but in some cases on-target adverse events may occur, including neurological symptoms. Ongoing and future studies will shed further light on the role and activity of TRK inhibitors in different patient groups, relation to other treatments, sequencing of TRK inhibitors as well as resistance mechanisms. 


\section{Acknowledgments}

Funding: The author has funding for research time by the Stockholm Cancer Society.

\section{Footnote}

Provenance and Peer Review: This article was commissioned by the Guest Editors (Silvia Novello, Francesco Passiglia) for the series "Looking for Chimeras in NSCLC: Widen Therapeutic Options Targeting Oncogenic Fusions" published in Translational Lung Cancer Research. The article has undergone external peer review.

Peer Review File: Available at http://dx.doi.org/10.21037/ tlcr-20-434

Conflicts of Interest: The author has completed the ICMJE uniform disclosure form (available at http:// dx.doi.org/10.21037/tlcr-20-434). The series "Looking for Chimeras in NSCLC: Widen Therapeutic Options Targeting Oncogenic Fusions" was commissioned by the editorial office without any funding or sponsorship. The author has no other conflicts of interest to declare.

Ethical Statement: The author is accountable for all aspects of the work in ensuring that questions related to the accuracy or integrity of any part of the work are appropriately investigated and resolved.

Open Access Statement: This is an Open Access article distributed in accordance with the Creative Commons Attribution-NonCommercial-NoDerivs 4.0 International License (CC BY-NC-ND 4.0), which permits the noncommercial replication and distribution of the article with the strict proviso that no changes or edits are made and the original work is properly cited (including links to both the formal publication through the relevant DOI and the license). See: https://creativecommons.org/licenses/by-nc-nd/4.0/.

\section{References}

1. Bray F, Ferlay J, Soerjomataram I, et al. Global cancer statistics 2018: GLOBOCAN estimates of incidence and mortality worldwide for 36 cancers in 185 countries. CA Cancer J Clin 2018;68:394-424.

2. Soda M, Choi YL, Enomoto M, et al. Identification of the transforming EML4-ALK fusion gene in non-small-cell lung cancer. Nature 2007;448:561-6.

3. Shaw AT, Ou SH, Bang YJ, et al. Crizotinib in ROS1rearranged non-small-cell lung cancer. $\mathrm{N}$ Engl J Med 2014;371:1963-71.

4. Valent A, Danglot G, Bernheim A. Mapping of the tyrosine kinase receptors trkA (NTRK1), trkB (NTRK2) and trkC(NTRK3) to human chromosomes 1q22, 9q22 and $15 \mathrm{q} 25$ by fluorescence in situ hybridization. Eur J Hum Genet 1997;5:102-4.

5. Amatu A, Sartore-Bianchi A, Bencardino K, et al. Tropomyosin receptor kinase (TRK) biology and the role of NTRK gene fusions in cancer. Ann Oncol 2019;30:viii5viii15.

6. Cocco E, Scaltriti M, Drilon A. NTRK fusion-positive cancers and TRK inhibitor therapy. Nat Rev Clin Oncol 2018;15:731-47.

7. Su YW, Zhou XF, Foster BK, et al. Roles of neurotrophins in skeletal tissue formation and healing. J Cell Physiol 2018;233:2133-45.

8. Garrido MP, Torres I, Vega M, et al. Angiogenesis in Gynecological Cancers: Role of Neurotrophins. Front Oncol 2019;9:913.

9. Minnone G, De Benedetti F, Bracci-Laudiero L. NGF and Its Receptors in the Regulation of Inflammatory Response. Int J Mol Sci 2017;18:1028.

10. Ricci A, Felici L, Mariotta S, et al. Neurotrophin and neurotrophin receptor protein expression in the human lung. Am J Respir Cell Mol Biol 2004;30:12-9.

11. Martin-Zanca D, Hughes SH, Barbacid M. A human oncogene formed by the fusion of truncated tropomyosin and protein tyrosine kinase sequences. Nature 1986;319:743-8.

12. Sartore-Bianchi A, Ardini E, Bosotti R, et al. Sensitivity to Entrectinib Associated With a Novel LMNA-NTRK1 Gene Fusion in Metastatic Colorectal Cancer. J Natl Cancer Inst 2015;108:djv306.

13. Milione M, Ardini E, Christiansen J, et al. Identification and characterization of a novel. Oncotarget 2017;8:55353-60.

14. Knezevich SR, McFadden DE, Tao W, et al. A novel ETV6-NTRK3 gene fusion in congenital fibrosarcoma. Nat Genet 1998;18:184-7.

15. Tognon C, Knezevich SR, Huntsman D, et al. Expression of the ETV6-NTRK3 gene fusion as a primary event in human secretory breast carcinoma. Cancer Cell 2002;2:367-76.

16. Skálová A, Vanecek T, Sima R, et al. Mammary analogue secretory carcinoma of salivary glands, containing the 
ETV6-NTRK3 fusion gene: a hitherto undescribed salivary gland tumor entity. Am J Surg Pathol 2010;34:599-608.

17. Stransky N, Cerami E, Schalm S, et al. The landscape of kinase fusions in cancer. Nat Commun 2014;5:4846.

18. Sinkevicius KW, Kriegel C, Bellaria KJ, et al. Neurotrophin receptor TrkB promotes lung adenocarcinoma metastasis. Proc Natl Acad Sci U S A 2014;111:10299-304

19. Okamura K, Harada T, Wang S, et al. Expression of TrkB and BDNF is associated with poor prognosis in non-small cell lung cancer. Lung Cancer 2012;78:100-6.

20. Odate S, Nakamura K, Onishi H, et al. TrkB/BDNF signaling pathway is a potential therapeutic target for pulmonary large cell neuroendocrine carcinoma. Lung Cancer 2013;79:205-14.

21. Vaishnavi A, Capelletti M, Le AT, et al. Oncogenic and drug-sensitive NTRK1 rearrangements in lung cancer. Nat Med 2013;19:1469-72.

22. Farago AF, Le LP, Zheng Z, et al. Durable Clinical Response to Entrectinib in NTRK1-Rearranged NonSmall Cell Lung Cancer. J Thorac Oncol 2015;10:1670-4.

23. Farago AF, Taylor MS, Doebele RC, et al. Clinicopathologic Features of Non-Small-Cell Lung Cancer Harboring an NTRK Gene Fusion. JCO Precis Oncol 2018;2018:PO.18.00037.

24. Lassen U. How I treat NTRK gene fusion positive cancers. ESMO Open 2019;4:e000612.

25. Solomon JP, Benayed R, Hechtman JF, et al. Identifying patients with NTRK fusion cancer. Ann Oncol 2019;30:viii16-22.

26. Gatalica Z, Xiu J, Swensen J, et al. Molecular characterization of cancers with NTRK gene fusions. Mod Pathol 2019;32:147-53.

27. Marchiò C, Scaltriti M, Ladanyi M, et al. ESMO recommendations on the standard methods to detect NTRK fusions in daily practice and clinical research. Ann Oncol 2019;30:1417-27.

28. Drilon A, Laetsch TW, Kummar S, et al. Efficacy of Larotrectinib in TRK Fusion-Positive Cancers in Adults and Children. N Engl J Med 2018;378:731-9.

29. Ardini E, Menichincheri M, Banfi P, et al. Entrectinib, a Pan-TRK, ROS1, and ALK Inhibitor with Activity in Multiple Molecularly Defined Cancer Indications. Mol Cancer Ther 2016;15:628-39.

30. Menichincheri M, Ardini E, Magnaghi P, et al. Discovery of Entrectinib: A New 3-Aminoindazole As a Potent Anaplastic Lymphoma Kinase (ALK), c-ros Oncogene 1
Kinase (ROS1), and Pan-Tropomyosin Receptor Kinases (Pan-TRKs) inhibitor. J Med Chem 2016;59:3392-408.

31. Lassen UN, Albert CM, Kummar S, et al. Larotrectinib efficacy and safety in TRK fusion cancer: an expanded clinical dataset showing consistency in an age and tumour agnostic approach. Ann Oncol 2018;29:viii133-48.

32. Hong DS, DuBois SG, Kummar S, et al. Larotrectinib in patients with TRK fusion-positive solid tumours: a pooled analysis of three phase $1 / 2$ clinical trials. Lancet Oncol 2020;21:531-40.

33. Demetri GD, Paz-Ares L, Farago AF, et al. Efficacy and safety of entrectinib in patients with NTRK fusion-positive tumours: pooled analysis of STARTRK-2, STARTRK-1, and ALKA-372-001. Ann Oncol 2018;29:ix173-8.

34. Doebele RC, Drilon A, Paz-Ares L, et al. Entrectinib in patients with advanced or metastatic NTRK fusionpositive solid tumours: integrated analysis of three phase 1-2 trials. Lancet Oncol 2020;21:271-82.

35. Hyman D, Kummar S, Farago F, et al. Phase I and expanded access experience of LOXO-195 (BAY 2731954), a selective next-generation TRK inhibitor (TRKi). Proceedings of the American Association for Cancer Research Annual Meeting 2019; 2019 Mar 29Apr 3; Atlanta, GA. Philadelphia (PA): AACR; Cancer Res 2019;79(13 Suppl):Abstract nr CT127.; 2019.

36. Drilon A, Moreno Garcia V, Patel J, et al. Efficacy and safety of larotrectinib in patients with tropomyosin receptor kinase (TRK) fusion lung cancer. Ann Oncol 2020;31:S754-840.

37. Drilon A, Paz-Ares L, Doebele RC, et al. Entrectinib in NTRK fusion-positive NSCLC: Updated integrated analysis of STARTRK-2, STARTRK-1 and ALKA-372001. Ann Oncol 2020;31:S462-504.

38. Russo M, Misale S, Wei G, et al. Acquired Resistance to the TRK Inhibitor Entrectinib in Colorectal Cancer. Cancer Discov 2016;6:36-44.

39. Drilon A, Li G, Dogan S, et al. What hides behind the MASC: clinical response and acquired resistance to entrectinib after ETV6-NTRK3 identification in a mammary analogue secretory carcinoma (MASC). Ann Oncol 2016;27:920-6.

40. Drilon A. TRK inhibitors in TRK fusion-positive cancers. Ann Oncol 2019;30:viii23-30.

41. Cocco E, Schram AM, Kulick A, et al. Resistance to TRK inhibition mediated by convergent MAPK pathway activation. Nat Med 2019;25:1422-7.

42. Fuse MJ, Okada K, Oh-Hara T, et al. Mechanisms of Resistance to NTRK Inhibitors and Therapeutic 
Strategies in NTRK1-Rearranged Cancers. Mol Cancer Ther 2017;16:2130-43.

43. Drilon A, Ou SI, Cho BC, et al. Repotrectinib (TPX0005) Is a Next-Generation ROS1/TRK/ALK Inhibitor That Potently Inhibits ROS1/TRK/ALK Solvent- Front Mutations. Cancer Discov 2018;8:1227-36.

44. Drilon A, Zhai D, Deng W, et al. Repotrectinib, a next generation TRK inhibitor, overcomes TRK resistance mutations including solvent front, gatekeeper and

Cite this article as: Ekman S. How selecting best therapy for metastatic NTRK fusion-positive non-small cell lung cancer? Transl Lung Cancer Res 2020;9(6):2535-2544. doi: 10.21037/tlcr$20-434$ compound mutations.: Proceedings of the American Association for Cancer Research Annual Meeting 2019; 2019 Mar 29-Apr 3; Atlanta, GA. Philadelphia (PA):

AACR; Cancer Res 2019;79(13 Suppl):Abstract nr 442.; 2019.

45. Tsakonas G, Ekman S. Oncogene-addicted non-small cell lung cancer and immunotherapy. J Thorac Dis 2018;10:S1547-55. 